\title{
ON THE EXISTENCE OF PERIODIC SOLUTIONS FOR SCALAR SECOND ORDER DIFFERENTIAL EQUATIONS WHEN ONLY THE ASYMPTOTIC BEHAVIOUR OF THE POTENTIAL IS KNOWN
}

\author{
ALESSANDRO FONDA \\ (Communicated by Charles Pugh)
}

\begin{abstract}
Assuming only asymptotic conditions on the potential function, we prove the existence of periodic solutions for equations whose nonlinearity stays below the first curve of Fučik's spectrum.
\end{abstract}

\section{INTRODUCTION AND STATEMENT OF THE RESULTS}

In this note we consider the periodic problem

$$
\left\{\begin{array}{l}
x^{\prime \prime}+g(x)=e(t), \\
x(0)-x(T)=x^{\prime}(0)-x^{\prime}(T)=0,
\end{array}\right.
$$

where $g: \mathbb{R} \rightarrow \mathbb{R}$ is a continuous function and $e:[0, T] \rightarrow \mathbb{R}$ is measurable and bounded. We define $G(x)=\int_{0}^{x} g(s) d s$, a primitive of $g(x)$, and $\bar{e}=$ $\frac{1}{T} \int_{0}^{T} e(t) d t$, the mean value of $e(t)$.

In our first result we assume the nonlinearity to lie, roughly speaking, between the first eigenvalue and the first curve of Fučik's spectrum.

Theorem 1. Assume that

$$
\mu:=\liminf _{x \rightarrow+\infty} \frac{2 G(x)}{x^{2}} \quad \nu:=\lim _{x \rightarrow-\infty} \frac{2 G(x)}{x^{2}}
$$

exist and are finite and positive. If

$$
\frac{\pi}{\sqrt{\mu}}+\frac{\pi}{\sqrt{\nu}}>T
$$

then problem $(\mathrm{P})$ has a solution.

Received by the editors September 21, 1991.

1991 Mathematics Subject Classification. Primary 34C25. 
As a consequence of Theorem 1, we have that, if the limit

$$
l:=\lim _{|x| \rightarrow \infty} \frac{2 G(x)}{x^{2}}
$$

exists and $0<l<\left(\frac{2 \pi}{T}\right)^{2}$, there exists a solution to $(\mathrm{P})$. It is not known whether such a result still holds when $l$ lies between any two eigenvalues of the associated linear operator.

Our second result deals with a one-sided assumption on the potential $G$. A further condition then has to be added in order to avoid resonance.

Theorem 2. Assume that

$$
\begin{aligned}
& \liminf _{x \rightarrow+\infty} \frac{2 G(x)}{x^{2}}<\left(\frac{\pi}{T}\right)^{2} \\
& \lim _{|x| \rightarrow \infty}(G(x)-\bar{e} x)=+\infty .
\end{aligned}
$$

Then problem (P) has a solution.

Notice that the above statements make use of assumptions relying only on the potential function $G$, and we do not require explicit conditions on the nonlinearity $g$, like monotonicity, sign conditions or growth restrictions (see [3-5, 8-10] and references therein).

The proof of Theorem 1 combines Leray-Schauder topological degree arguments with the upper and lower solutions method. To prove Theorem 2 we also need a variational setting. We will develop some ideas from Gossez and Omari [8, 9], Fernandes and Zanolin [5], and De Figueireido and Ruf [2].

In $\S 3$, we will also state a necessary and sufficient nonresonance condition for problem (P), when assumption (i) of Theorem 2 holds. Needless to say, the symmetrical versions of our results hold as well, the assumptions at $\pm \infty$ being interchangeable.

Without loss of generality, we will consider from now on the case $\bar{e}=0$.

\section{Proof of Theorem 1}

We first remark that, since $\mu$ and $\nu$ are positive, the function $g$ is unbounded from below and from above on $\mathbb{R}$. Since $e$ is bounded, there exist $A$ and $B$ in $\mathbb{R}$ such that, for a.e. $t \in[0, T]$,

$$
g(A)<e(t)<g(B) .
$$

We will use some arguments from [8]. If $A>B$, the result is a consequence of the lower and upper solutions method (cf. [1,8]). Assume then $A<B$. Replacing $x$ by $x-\frac{A+B}{2}$, it is of no loss of generality to suppose

$$
A<0<B \text {. }
$$

Moreover, we can assume the existence of a constant $c_{1}>0$ such that, for every $s \in \mathbb{R}$,

$$
\operatorname{sgn}(s) g(s)>-c_{1} \text {. }
$$

In fact, otherwise one could find $A^{\prime}>B$ with the same property as $A$, and the lower and upper solutions method would apply again. 
Now we fix a $\theta \in] 0,\left(\frac{\pi}{T}\right)^{2}[$ and we consider, for $\lambda \in] 0,1[$, the problem

$$
\left\{\begin{array}{l}
x^{\prime \prime}+\lambda \theta x+(1-\lambda) g(x)=(1-\lambda) e(t) \\
x(0)-x(T)=x^{\prime}(0)-x^{\prime}(T)=0
\end{array}\right.
$$

The existence of a solution to $(P)$ will be assured by the Leray-Schauder theory if we are able to find an open bounded subset $\mathscr{A}$ of the space $C(0, T)$, containing 0 , whose boundary does not contain any of the solutions of $\left.\left(\mathbf{P}_{\lambda}\right), \lambda \in\right] 0,1[$ (cf. [10]). We claim that there exist two constants $R$ and $S$ such that $R<$ $A<B<S$, and the set

$$
\mathscr{A}=\{x \in C(0, T) \mid \min (x) \in] R, B[, \max (x) \in] A, S[\}
$$

is the one we are looking for. The boundary of set $\mathscr{A}$ is

$$
\begin{aligned}
\partial \mathscr{A}=\{x \in C(0, T) \mid & \min (x) \in[R, B], \max (x) \in[A, S] \text { and } \\
& \text { either } \min (x) \in\{R, B\} \text { or } \max (x) \in\{A, S\}\} .
\end{aligned}
$$

If $x$ is a solution of $\left(\mathrm{P}_{\lambda}\right)$, it cannot be that $\min (x)=B$ or $\max (x)=A$. This can easily be seen by writing the equation in $\left(\mathbf{P}_{\lambda}\right)$ at the points where $x$ attains its minimum and maximum and taking into account (1) and (2).

Notice that, if $x \in \partial \mathscr{A}$,

$$
\exists t_{x} \in[0, T]: x\left(t_{x}\right) \in[A, B]
$$

We will find $S>B$ such that $\max (x) \neq S$ for every solution $x$ of $\left(\mathrm{P}_{\lambda}\right)$ having the property (4). Take $\mu^{\prime}>\mu$ in such a way that $\left(\pi / \sqrt{\mu^{\prime}}\right)+(\pi / \sqrt{\nu})>T$, and define $\hat{e}(t)=e(t)+c_{1}, \hat{g}(x)=g(x)+c_{1}$, and correspondingly $\widehat{G}(x)=$ $G(x)+c_{1} x$. Then it is easy to see that

$$
\underset{x \rightarrow+\infty}{\limsup }\left[\mu^{\prime} \frac{x^{2}}{2}-\widehat{G}(x)\right]=+\infty
$$

and we can find a sequence $\left(S_{n}\right)$ such that $S_{n} \rightarrow+\infty$ as $n \rightarrow \infty$ and

$$
\forall s \in\left[0, S_{n}\left[, \quad \mu^{\prime} \frac{s^{2}}{2}-\widehat{G}(s)<\mu^{\prime} \frac{S_{n}^{2}}{2}-\widehat{G}\left(S_{n}\right) .\right.\right.
$$

We will show that we can take $S=S_{\bar{n}}$ for $\bar{n}$ large enough. In fact, suppose by contradiction that there exist two sequences $\left(x_{n}\right),\left(\lambda_{n}\right)$ such that $\left.\lambda_{n} \in\right] 0,1[$, $x_{n}$ is a solution of $\left(\mathrm{P}_{\lambda_{n}}\right), \max \left(x_{n}\right)=S_{n}$, and $\exists t_{n} \in[0, T]: x_{n}\left(t_{n}\right) \in[A, B]$.

First we prove that $m_{n}:=\min \left(x_{n}\right) \rightarrow-\infty$ as $n \rightarrow \infty$. Suppose by contradiction that, for a subsequence, $\left(m_{n}\right)$ is bounded from below. Then, by (3), there is a constant $c_{2}>0$ such that

$$
\lambda_{n} \theta x_{n}(t)+\left(1-\lambda_{n}\right) g\left(x_{n}(t)\right)>-c_{2}
$$

for every $t \in[0, T]$. Since

$$
\int_{0}^{T}\left[\lambda_{n} \theta x_{n}+\left(1-\lambda_{n}\right) g\left(x_{n}\right)\right]=0
$$


multiplying the equation in $\left(\mathrm{P}_{\lambda_{n}}\right)$ by $\tilde{x}_{n}=x_{n}-\frac{1}{T} \int_{0}^{T} x_{n}(t) d t$ and integrating, we get

$$
\begin{aligned}
\int_{0}^{T}\left(\tilde{x}_{n}^{\prime}\right)^{2} & =\int_{0}^{T}\left[\lambda_{n} \theta x_{n}+\left(1-\lambda_{n}\right) g\left(x_{n}\right)+c_{2}\right] \tilde{x}_{n}-\int_{0}^{T}\left[c_{2}+\left(1-\lambda_{n}\right) e\right] \tilde{x}_{n} \\
& \leq\left\|\tilde{x}_{n}\right\|_{L^{\infty}}\left\{\int_{0}^{T}\left[\lambda_{n} \theta x_{n}+\left(1-\lambda_{n}\right) g\left(x_{n}\right)+c_{2}\right]+c_{2} T+\|e\|_{L^{1}}\right\} \\
& \leq\left\|\tilde{x}_{n}\right\|_{L^{\infty}}\left[2 c_{2} T+\|e\|_{L^{1}}\right] .
\end{aligned}
$$

Then, $\left(\tilde{x}_{n}\right)$ is bounded, and this contradicts the fact that $\max \left(x_{n}\right) \rightarrow+\infty$ and $\exists t_{n} \in[0, T]: x_{n}\left(t_{n}\right) \in[A, B]$. This proves that $m_{n} \rightarrow-\infty$ as $n \rightarrow \infty$.

Extending our functions by T-periodicity, we can then find two intervals $\left[\alpha_{n}, \beta_{n}\right]$ and $\left[\gamma_{n}, \delta_{n}\right]$ containing, respectively, a point of minimum and a point of maximum of $x_{n}$, such that

$$
\begin{gathered}
x_{n}\left(\alpha_{n}\right)=x_{n}\left(\beta_{n}\right)=x_{n}\left(\gamma_{n}\right)=x_{n}\left(\delta_{n}\right)=0, \\
\left.x_{n}(t)<0 \text { for } t \in\right] \alpha_{n}, \beta_{n}[, \\
\left.x_{n}(t)>0 \text { for } t \in\right] \gamma_{n}, \delta_{n}[, \\
\left(\beta_{n}-\alpha_{n}\right)+\left(\delta_{n}-\gamma_{n}\right) \leq T .
\end{gathered}
$$

We will now use some ideas from $[5,6,11]$. We first restrict our analysis to the interval $\left[\gamma_{n}, \delta_{n}\right]$. Because of (3), we have that $\hat{g}(s)>0$ for all $s>0$. We can define, for $S>0$, the time-map

$$
\hat{\tau}(S)=\sqrt{2} \int_{0}^{S} \frac{d \xi}{\sqrt{\widehat{G}(S)-\widehat{G}(\xi)}} .
$$

It has been shown in [6] that the time-map $\hat{\tau}\left(S_{n}\right)$ is a good estimate, as $n \rightarrow \infty$, for the length of $\left[\delta_{n}, \gamma_{n}\right]$. Since, by $(5), \hat{\tau}\left(S_{n}\right) \geq\left(\pi / \sqrt{\mu^{\prime}}\right)$ for every $n$, we obtain

$$
\liminf _{n \rightarrow \infty}\left(\delta_{n}-\gamma_{n}\right) \geq \frac{\pi}{\sqrt{\mu^{\prime}}} .
$$

On the other hand, one can estimate the length of $\left[\alpha_{n}, \beta_{n}\right]$ in the following way. One defines $\check{e}(t)=e(t)-c_{1}, \check{g}(x)=g(x)-c_{1}$, and correspondingly $\check{G}(x)=G(x)-c_{1} x$. Then, for $m<0$, the time-map is defined by

$$
\check{\tau}(m)=\sqrt{2} \int_{m}^{0} \frac{d \xi}{\sqrt{\check{G}(m)-\check{G}(\xi)}} .
$$

Using the fact that $\nu$ is the limit of the quotient $2 G(x) / x^{2}$, one can prove that

$$
\liminf _{m \rightarrow-\infty} \check{\tau}(m) \geq \frac{\pi}{\sqrt{\nu}}
$$

(cf. [11, Corollary 8]). Since $\check{\tau}\left(m_{n}\right)$ is a good estimate, as $n \rightarrow \infty$, for the length of $\left[\alpha_{n}, \beta_{n}\right]$, we have that

$$
\liminf _{n \rightarrow \infty}\left(\beta_{n}-\alpha_{n}\right) \geq \frac{\pi}{\sqrt{\nu}} .
$$


We get a contradiction with (7) and the fact that $\left(\pi / \sqrt{\mu^{\prime}}\right)+(\pi / \sqrt{\nu})>T$. So we can choose $S=S_{\bar{n}}$, with $\bar{n}$ large enough.

Finally, we prove that there is $R<A$ such that, if $x$ is a solution of $\left(\mathrm{P}_{\lambda}\right), \lambda \in$ ]0, 1[ , satisfying $\max (x) \leq S$ and (4), then $\min (x)>R$. In fact, if $\max (x) \leq$ $S$, there exists a constant $c_{3}>0$ such that

$$
\lambda \theta x(t)+(1-\lambda) g(x(t))<c_{3} .
$$

Multiplying the equation in $\left(\mathbf{P}_{\lambda}\right)$ by $\tilde{x}$ and integrating, we obtain an estimate analogous to (6). Hence $\|\tilde{x}\|_{L^{\infty}}$ is bounded by a constant depending only on $S$, and since $x$ satisfies (4), there is $R<A$ such that $\min (x)>R$.

Thus we showed that no solution of $\left.\left(\mathrm{P}_{\lambda}\right), \lambda \in\right] 0,1[$, can lie on $\partial \mathscr{A}$, and the proof is complete.

\section{Proof of Theorem 2}

If $g$ is unbounded from above and below on $\mathbb{R}$, one can proceed as in the proof of Theorem 1. The only difference lies in the contradiction with (7), which is already reached in (8), since $\mu^{\prime}<\left(\frac{\pi}{T}\right)^{2}$. In the spirit of [8], we then have the following.

Corollary 1. Assuming that

$$
\liminf _{x \rightarrow+\infty} \frac{2 G(x)}{x^{2}}<\left(\frac{\pi}{T}\right)^{2}
$$

problem (P) has a solution for every $e \in L^{\infty}(0, T)$ if and only if $g$ is unbounded from above and below.

Now assume $g$ to be bounded either from below or above; let us treat the first case, the other being similar. So, assume there exists $c_{4}>0$ such that, for all $s \in \mathbb{R}$,

$$
g(s) \geq-c_{4}
$$

We will prove the existence of a solution for $(\mathrm{P})$ by finding a critical point of the associated functional $\phi: H_{T}^{1} \rightarrow R$ defined as

$$
\phi(x)=\int_{0}^{T}\left[\frac{1}{2}\left(x^{\prime}(t)\right)^{2}-G(x(t))+e(t) x(t)\right] d t .
$$

We will show that the functional $\phi$ has a mountain-pass geometry and satisfies the Palais-Smale condition.

Define the set $\mathscr{S}=\left\{x \in H_{T}^{1}: x \leq 0\right.$ and $\left.\exists t_{x} \in[0, T]: x\left(t_{x}\right)=0\right\}$. Extending our functions by periodicity, for every $x \in \mathscr{S}$ we can write $\phi(x)$ as an integral over the interval $\left[t_{x}, t_{x}+T\right]$. Because of (9), we have that $\lim _{x \rightarrow-\infty} \frac{2 G(x)}{x^{2}}=0$, and it is easy to see, by Poincaré's inequality, that $\phi$ is bounded below on $\mathscr{S}$. On the other hand, (ii) implies that $(-\phi)$ is coercive on the space of constant functions. We can then find $r>0$ sufficiently large 
for which

$$
\max \{\phi(-r), \phi(r)\}<\inf _{\mathscr{S}} \phi .
$$

Since, by continuity, every path in $\Gamma=\left\{\gamma \in C\left([-r, r], H_{T}^{1}\right): \gamma( \pm r)= \pm r\right\}$ has to cross $\mathscr{S}$, we have

$$
\inf _{\mathscr{S}} \phi \leq \inf \sup _{\Gamma} \phi .
$$

We are then in a mountain-pass geometry.

In order to conclude, we now prove the Palais-Smale condition. Let $\left(x_{n}\right)$ be a sequence in $H_{T}^{1}$ such that $\left(\phi\left(x_{n}\right)\right)$ is bounded and $\phi^{\prime}\left(x_{n}\right) \rightarrow 0$, as $n \rightarrow \infty$. Then there is a constant $c_{5}>0$ such that, for every $u \in H_{T}^{1}$,

$$
\left|\int_{0}^{T}\left[x_{n}^{\prime} u^{\prime}-g\left(x_{n}\right) u+e u\right] d t\right|<c_{5}\|u\|_{H^{1}} .
$$

Taking $u \equiv 1$, we get

$$
\left|\int_{0}^{T} g\left(x_{n}(t)\right) d t\right| \leq c_{5} \sqrt{T}
$$

hence, by (9),

$$
\int_{[g \geq 0]} g\left(x_{n}(t)\right) d t \leq c_{5} \sqrt{T}-\int_{[g<0]} g\left(x_{n}(t)\right) d t \leq c_{5} \sqrt{T}+c_{4} T .
$$

Consequently, there is a constant $c_{6}$ such that

$$
\int_{0}^{T}\left|g\left(x_{n}(t)\right)\right| d t \leq c_{6} .
$$

Taking $u=\tilde{x}_{n}$ in (10) permits us to conclude that $\left(\tilde{x}_{n}\right)$ has to be bounded. Assume by contradiction that, for a subsequence, $\left\|x_{n}\right\|_{H^{1}} \rightarrow \infty$. Then $\left|x_{n}(t)\right| \rightarrow$ $\infty$ uniformly in $t$. But this is in contradiction with the fact that $\left(\tilde{x}_{n}\right)$ and $\left(\phi\left(x_{n}\right)\right)$ are bounded, since $G$ is coercive. So $\left(x_{n}\right)$ is bounded, and the PalaisSmale condition holds (see [12]).

\section{REFERENCES}

1. A. Adje, Sur et sous solutions dans les équations différentielles discontinues avec conditions aux limites non linéaires, Ph.D. Thesis, Louvain-la-Neuve, 1987.

2. D. de Figueiredo and B. Ruf, On a superlinear Sturm-Liouville equation and a related bouncing problem, J. Reine Angew. Math. 421 (1991), 1-22.

3. T. Ding, R. Iannacci, and F. Zanolin, Existence and multiplicity results for periodic solutions of semilinear Duffing equations, J. Differential Equations (to appear).

4. T. Ding and F. Zanolin, Time-maps for the solvability of periodically perturbed nonlinear Duffing equations, Nonlinear Anal. TMA 17 (1991), 635-653.

5. M. L. C. Fernandes and F. Zanolin, Periodic solutions of a second order differential equation with one-sided growth restrictions or the restoring term, Arch. Math. 51 (1988), 151-163.

6. A. Fonda and F. Zanolin, On the use of time-maps for the solvability of nonlinear boundary value problems, Arch. Math. 59 (1992), 245-259.

7. S. Fučik, Solvability of nonlinear equations and boundary value problems, Reidel, Dortrecht, 1980.

8. J.-P. Gossez and P. Omari, Periodic solutions of a second order ordinary differential equation: a necessary and sufficient condition for nonresonance, J. Differential Equations 94 (1991), 67-82. 
9. $\longrightarrow$ A note on periodic solutions for a second order ordinary differential equation, Boll. Un. Mat. Ital. A 5 (1991), 223-231.

10. J. Mawhin, Points fixes, point critiques et problèmes aux limites, Sem. Math. Sup., vol. 92 , Presses Univ. Montreal, Montreal, 1985.

11. Z. Opial, Sur les périodes des solutions de l'équation differentielle $x^{\prime \prime}+g(x)=0$, Ann. Polon. Math. 10 (1961), 49-72.

12. P. H. Rabinowitz, Minimax methods in critical point theory with applications to differential equations, CBMS Regional Conf. Ser. in Math., vol. 65, Amer. Math. Soc., Providence, RI, 1986.

Department of Mathematics, University of Trieste, P. le Europa 1, 34100 Trieste, ITALY

E-mail address: fondass@univ.trieste.it (bitnet) 